\title{
Variación de la percepción del recurso paisaje en el sur de Chile
}

\author{
Variation of the perception of the landscape resource in Southern Chile
}

\author{
ANDRES MUÑOZ-PEDREROS ${ }^{1}$, JUAN MONCADA-HERRERA ${ }^{2} \&$ ALBERTO LARRAIN ${ }^{3}$ \\ ${ }^{1}$ Departamento de Ciencias Ambientales y Químicas. Facultad de Ciencias. Universidad Católica de \\ Temuco, Casilla 15-D, Temuco, Chile, e-Mail: amunoz@uctem.cl \\ ${ }^{2}$ Departamento de Matemáticas y Computación. Facultad de Ciencias. Universidad Católica de Temuco, \\ Casilla 15-D, Temuco, Chile; ${ }^{3}$ Facultad de Ciencias Naturales y Oceanográficas. Universidad de \\ Concepción, Casilla 160-C, Concepción
}

\begin{abstract}
RESUMEN
El paisaje es el escenario de la actividad humana, por lo que cualquier acción artificial repercute inmediatamente en los factores perceptuales. Diversos autores defienden la idea de universalidad de las preferencias estéticas, lo que es refutado por quienes consideran determinantes los aspectos culturales e idiosincráticos a la hora de apreciar paisajes. Pareciera que una posición intermedia es, por ahora, la más atractiva. El objetivo de esta investigación es establecer la variabilidad derivada del evaluador y proponer técnicas que controlen, lo más posible, esta fuente de variación. Hemos seleccionado tres paneles, uno caracterizado como exigentes en la evaluación de paisaje, el segundo como transformadores y un tercero como control. De trabajos sincrónicos se evaluaron 144 paisajes, principalmente con bosque nativo, actividades ganaderas, agrícolas y silvícolas y ambientes lacustres. Se aplicó una encuesta cuya información fue dispuesta en arreglos matriciales de acuerdo a la naturaleza de los análisis que de ella se hicieron. Las técnicas estadísticas consideraron recursos descriptivos, tanto gráficos como numéricos y técnicas de análisis multivariado, particularmente análisis en componentes principales. Se estableció la variabilidad intragrupo de los observadores, detectándose diferencias significativas en algunos subgrupos que lo componen. Respecto a la variabilidad intergrupo de los observadores, se constató una alta coherencia interna en los tres grupos, la que varía según las macrounidades de paisaje. Se recomiendan criterios para la constitución de un panel que disminuya la subjetividad para evaluar paisaje del sur de Chile.
\end{abstract}

Palabras clave: evaluación de paisaje, paisaje visual, actividades rurales.

\begin{abstract}
The landscape is the stage of human activity, in which any artificial action rebounds immediately on the perception. Various authors defend the idea of the universality of aesthetic preferences, an idea that is refuted by those who consider cultural and idiosyncratic features as determinant of peoples appreciation of landscapes. It seems that an intermediate position is, for the time being, the most attractive. The objectives of this investigation is to establish the variability derived from the evaluator and to propose techniques that are effective in controling this source of variation. We selected three panels, one characterized as "demanding" in the evaluation of a landscape, the second as "transformers" and a third one as a control. One hundred and forty-four (144) landscapes were evaluated from synchronized investigations, mainly with native forest, cattle, agricultural and forest activities, and lake environments. A survey was set up with the information organized as matrix arrangements according to the nature of the analysis from where it came. The statistical techniques considered descriptive resources (graphics as well as numeric), test of multivariance analysis, and particularly principal components analysis. Intragroup variability of the observers was established, with the detect on of significant differences in some the subgroups that compose them. With reference to intergroup variability of the observers, a high internal coherence in the three groups was found, which varied according to the macrounits of the landscape. Criteria for the constitution of a panel that diminishes the subjectivity to evaluate landscapes of the Southern chilean region are recommended.
\end{abstract}

Key words: Evaluation of landscape, visual landscape, rural activities.

\section{INTRODUCCION}

Los paisajes pueden clasificarse en: naturales y artificiales o construídos. A su vez los naturales pueden subclasificarse como intervenidos y no intervenidos $\mathrm{y}$, ultimamente, como contínuos o fragmentados. El paisaje puede identificarse, como el conjunto de interrelaciones derivadas de la interacción entre geomorfología, clima, vegetación, fauna, agua y modificaciones antrópicas (MOPT 1993). Bernáldez (1981) lo define como la percepción plurisensorial de un sistema de 
relaciones ecológicas. En esta multidimensionalidad radica la dificultad de su estudio (Galiano \& Abello 1984). De este modo, las restricciones técnicas y de escalas sólo permiten considerar (por ahora) sus valores visuales. Por lo tanto se buscan métodos confiables para establecer la calidad visual de un paisaje. El concepto de paisaje visual, considera la estética y la capacidad de percepción que del paisaje tiene un observador. El énfasis se pone en el efecto de un paisaje determinado sobre el observador, $y$, aunque intervienen los cinco sentidos, el visual es el más relevante. Así, las fuentes fundamentales de variabilidad del paisaje visual son: a) la derivada del propio territorio y b) la derivada del observador. En este estudio, nos interesamos por la segunda fuente de variación: el observador.

Appleton (1981), Kaplan \& Kaplan (1982) y Bernáldez (1981) proponen que las preferencias por ciertos paisajes tendrían una explicación evolutiva al asociar las inclinaciones estéticas con ciertos ambientes que aumentan la capacidad de supervivencia del individuo. Diversos autores (e.g., Kaplan 1976, Ruiz et al. 1990¹) defienden la idea de universalidad de las preferencias estéticas. Es decir los seres humanos en general, prefieren ciertos paisajes. Contradice esta idea Jacques (1980), que considera determinantes los aspectos culturales e idiosincráticos a la hora de apreciar paisajes. Pareciera que una posición intermedia es, por ahora, la más atractiva. Es decir, existiría una preferencia general del ser humano por ciertos paisajes reconociendo un sustrato darwiniano asociado a la supervivencia, pero estas "preferencias innatas" estarían influenciadas culturalmente. Es, entonces, tarea inmediata detectar esas variaciones e intentar su eventual control para hacer de la evaluación de paisajes, una herramienta confiable que lleve a resultados comparables.

El desarrollo de técnicas, para evaluar los paisajes, permitirá tomar decisiones ambientales, respecto a este recurso, con bases sólidas. De este modo, metodologías adaptadas a las características culturales de un país, las harán instrumentos fiables de gestión ambiental. El objetivo de esta investigación es establecer la variabilidad derivada del evaluador y proponer técnicas que controlen, lo más posible, esa fuente de variación.

\footnotetext{
I Ruiz JP, J Banayas, JV De Luicio, C López, FG Bernaldez \& J Aranguren (1990) Comparaciones interculturales de preferencias paisjísticas. I Congreso de Ciencias del Paisaje. Barcelona
}

\section{MATERIALES Y METODOS}

\section{Selección de los paneles de evaluación}

En teoría, la población total del país es un potencial observador de paisajes, sin embargo no todos tienen la posibilidad de ser, directa o indirectamente, gestores en un paisaje. Para el paisaje rural acotamos los potenciales evaluadores a personas con estudios universitarios completos y vinculados directa o indirectamente con el espacio rural. De este modo hemos seleccionado un panel conformado por tres grupos: El primero constituído por personas vinculadas a la actividad turística y recreacional, naturalistas y ambientalistas. Todos ellos caracterizados como exigentes en la evaluación de paisaje. El segundo, integrado por personas vinculados a la actividad agrícola, ganadera y silvícola (Ingenieros Forestales, Ingenieros Agrónomos y Médicos Veterinarios), caracterizados como transformadores de paisaje rural. El tercer grupo estuvo integrado por profesionales con adiestramiento básico en botánica, geomorfología, evaluación de recursos naturales y en especial de paisajes, quienes actuaron como grupo control. Con los paneles constituidos y los paisajes evaluados se realizó un análisis de la variabilidad del observador, es decir se estableció la variabilidad en la evaluación de los distintos panelistas frente a un mismo paisaje.

\section{Selección de imágenes y evaluación}

Se tomaron imágenes con una cámara reflex dotada de un gran angular de $28 \mathrm{~mm}$, en un transecto de $587 \mathrm{~km}$ a lo largo de la ruta 5 sur de Chile, en el tramo comprendido entre Cabrero $\left(37^{\circ} 00^{\circ} \mathrm{S}\right.$, $\left.72^{\circ} 23^{\circ} \mathrm{O}\right)$ y Puerto Montt $\left(41^{\circ} 30^{\circ} \mathrm{S}, 72^{\circ} 50^{\circ} \mathrm{O}\right)$. El transecto se escogió por la integración de tres variables: a) concentración de asentamientos humanos, b) accesibilidad y c) flujo de pasajeros y turistas. En este transecto se registraron todas las unidades de paisaje (divisiones espaciales que cubren el territorio a estudiar, sensu Escribano et al. (1991) y entendida como una agregación ordenada y coherente de las partes elementales de un paisaje. Por las características de algunas unidades de paisaje seguimos a Ramírez et al. (1992) en las definiciones de bosques, formación de parques, matorrales y praderas. El transecto se recorrió en el verano de 1997. Para determinar una unidad de paisaje se tomó como componente central la cubierta vegetal, que es el componente de paisaje más representativo en el área de estudio, agregándose el relieve para disgregarla en 48 subunidades de paisaje para toda el área de estu- 
dio, las que a su vez se agruparon en seis macrounidades: bosque nativo, cultivos agrícolas, plantaciones exóticas, salto del Laja, río Cautín y obstrucciones visuales. Estas últimas impiden al observador percibir el paisaje. Estas obstrucciones están en un primer plano, de modo tal que interceptan la visión y según su composición se han agrupado en tres tipos: matorrales, arboledas y cortes viales. De este modo se presentaron, al panel de observadores, 48 paisajes, cada uno de ellos con tres réplicas (total 144 imágenes)

El método de evaluación es mixto con valoración directa de subjetividad representativa y análisis posterior indirecto con análisis de componentes propuesto por Muñoz-Pedreros (1993) y que considera el uso de diapositivas y la aplicación de un instrumento de evaluación. Usar sustitutos del paisajes introduce una variabilidad notable, ya que no es lo mismo mostrar un paisaje al natural que una fotografía o un dibujo. Pese a esto, muchos autores han estudiado y encontrado correlación entre los paisajes naturales y las diapositivas, incluso fotografías (véase a Dunn 1974, Daniel \& Boster 1976, Jackson 1978, Savolainen \& Kellomäki 1984).

La estandarización de las imágenes siguió a Litton (1972), Weddle (1973), De Veer \& Burrogh (1978) y MOPT (1993). El instrumento para evaluar fue una encuesta que contenía dos partes, la primera fue un cuestionario estructurado que permitió caracterizar a los evaluadores según estratificación etaria, sexo y actividad profesional y la segunda una lista de adjetivos jerarquizados en la idea de Craik (1975) y adaptados por MuñozPedreros et al. (1993). Los evaluadores no conocian las imágenes, el instrumento de evaluación ni la metodología a emplear, los que fueron capacitados inmediatamente antes de la evaluación, por lo que no existian critérios preestablecidos. Las valoraciones se realizaron mostrando las diapositivas a los paneles de evaluadores con una cadencia de 20 segundos cada una y los evaluadores marcaron el calificativo, en la encuesta, que les pareció mejor descriptor. Los calificativos fueron transformados a la escala numérica de modo que cada unidad de paisaje recibió una valoración, la que teoricamente varió entre 1 y 30.

\section{Análisis de la información}

La información obtenida, a partir de las evaluaciones que los distintos observadores hicieron de las imágenes presentadas, fue dispuesta en matrices de acuerdo a la naturaleza de los análisis que de ella se hicieron. La naturaleza de la informa- ción posibilitó análisis en dos direcciones: una orientada a la evaluación de los observadores de los distintos paisajes, y otra que permitió un análisis de los paisajes, en relación a sus características propias y a la percepción que de ellos tienen los distintos evaluadores. En un caso estuvimos interesados en levantar, en la medida que los datos lo permitan, una tipología de los evaluadores, mientras que en el otro caso fijamos nuestro interés en una tipología de los paisajes. Cabe mencionar que este último objetivo es secundario, ya que el objetivo principal de la investigación fue describir la variabilidad de los evaluadores. En síntesis, se realizó una descripción general de la información, un análisis y estudio de la variabilidad de los observadores, y un estudio sobre una eventual tipología de los observadores.

Las técnicas estadísticas consideraron recursos descriptivos, tanto gráficos como numéricos y técnicas de análisis multivariado (JohnsonWichern 1988, Chatfield-Collins 1992, Crivisqui 1993). Se utilizó Análisis de la Varianza (multifactorial y univariado) para estudiar y/o describir la variabilidad de los observadores en la evaluación de los paisajes, análisis de componentes principales para identificar las eventuales tipologías, utilizando elementos suplementarios para darle mayor alcance a las interpretaciones y construcciones que puedan obtenerse. El coeficiente de variación (CV) se uso como medida de homogeneidad de los grupos de evaluadores. El procesamiento de la información se realizó con los programas estadísticos Statgraphics $6.0 \mathrm{y}$ SPAD 3.5.

\section{RESULTADOS Y DISCUSION}

La variabilidad de los observadores se apreció desde dos perspectivas. Por un lado, se determinó la variabilidad intragrupo de los observadores, es decir se estableció la coherencia interna de cada grupo de observadores. Por otro lado, se estableció la variabilidad intergrupo de los observadores, es decir, se estudiaron las diferencias entre los diferentes grupos de observadores.

\section{Variabilidad intragrupo de los observadores}

En la Tabla 1 se muestran las principales características de resumen del grupo transformadores. Aparecen aquí como más homogéneos el subgrupo Ingenieros Forestales (coeficiente de variación de $25,2 \%$ ). Por otro lado esta coherencia interna se manifiesta más claramente en la macrounidad 
TABLA 1

Variación de la evaluación del paisaje en el grupo de transformadores en un transecto (Cabrero-Puerto Montt) del sur de Chile. 1997. DE= Desviación Estándar, CV= Coeficiente de Variación

Variation of the landscape evaluation in the transformer groups in a Southern Chilean transect (Cabrero-Puerto Montt). 1997. $\mathrm{DE}=$ Standard Deviation, $\mathrm{CV}=$ Coeficcient of variation

\begin{tabular}{lcccrcrcrc}
\hline & \multicolumn{3}{c}{$\begin{array}{c}\text { Ingenieros } \\
\text { Forestales }\end{array}$} & \multicolumn{3}{c}{$\begin{array}{c}\text { Ingenieros } \\
\text { Agrónomos }\end{array}$} & \multicolumn{3}{c}{$\begin{array}{c}\text { Médicos } \\
\text { Veterinarios }\end{array}$} \\
Macrounidad & Promedio & DE & CV & Promedio & DE & CV & Promedio & DE & CV \\
\hline Bosque Nativo & 18,5 & 3,4 & 18,4 & 15,9 & 4,9 & 30,6 & 13,6 & 2,4 & 17,8 \\
Cultivos Agrícolas & 16,0 & 6,2 & 38,8 & 10,1 & 4,1 & 40,9 & 10,6 & 3,3 & 31,0 \\
Plantaciones Exóticas & 12,9 & 3,2 & 24,8 & 6,1 & 3,2 & 53,1 & 8,3 & 3,3 & 39,2 \\
Río Cautín & 16,4 & 2,8 & 17,1 & 10,7 & 4,6 & 42,8 & 14,5 & 2,8 & 19,5 \\
Salto del Laja & 22,1 & 4,8 & 21,8 & 21,5 & 5,7 & 26,3 & 19,6 & 7,1 & 36,3 \\
Obstrucciones & 11,6 & 4,5 & 38,8 & 8,4 & 5,0 & 59,1 & 10,0 & 3,3 & 33,3 \\
& & & & & & & & & \\
Total paisajes & 16,3 & 4,1 & 25,2 & 12,1 & 4,6 & 38,0 & 12,8 & 3,7 & 28,9 \\
\hline
\end{tabular}

río Cautín. Además, este subgrupo evalúa con mayores ponderaciones a paisajes de la macrounidad salto del Laja, mientras que los paisajes de más baja valoración son los de la macrounidad obstrucciones visuales, seguidos por los de plantaciones exóticas.

Los Ingenieros Agrónomos, por su parte, son los que muestran la menor coherencia interna con un coeficiente de variación de un $38 \%$. A modo de ejemplo, las macrounidades de paisaje obstrucciones y plantaciones exóticas presentan un coeficiente de variación mayor que el $50 \%$, sin embargo coincide con los paisajes de más baja valoración. Por el contrario, en los paisajes mejor evaluados (e.g., salto del Laja) la dispersión relativa es notablemente menor $(\mathrm{CV}=26,3 \%)$.

Finalmente hay que hacer notar que el subgrupo Médicos Veterinarios posee una coherencia interna intermedia entre los grupos anteriores con un coeficiente de variación de $28,9 \%$. La coherencia interna aumenta notoriamente en las macrounidades bosque nativo y río Cautín $(\mathrm{CV}=$ $17,8 \%$ y $\mathrm{CV}=19,5 \%$ respectivamente). La dispersión relativa es mayor en el paisaje plantaciones exóticas (CV $=29,2 \%$ ), considerado, además, el peor evaluado (promedio $=8,3$ ).

De un análisis estadístico de las diferencias observadas en la Tabla 1 , se concluye que en el grupo transformadores hay diferencias significativas en los subgrupos que lo componen $(\mathrm{P}<$ 0,0005 ). Dentro de los tres subgrupos, las mayores diferencias, según la prueba de Tukey-HSD, se dan entre el subgrupo Ingenieros Forestales y el subgrupo Médicos Veterinarios y entre Ingenieros Forestales e Ingenieros Agrónomos. Es decir agrónomos y veterinarios perciben de modo similar los paisajes, diferenciándose significativamente (a un nivel de 0,05 ) de los forestales. Esto es evidente para los paisajes de bosque nativo, cultivos y plantaciones (Tabla 2). No existen diferencias significativas para los paisajes río Cautín y salto del Laja.

En el grupo de exigentes no se detectaron diferencias significativas globales en los subgrupos que lo componen $(\mathrm{P}=0,3008)$. Sin embargo, al realizar los análisis de cada macrounidad de paisaje, se detectaron diferencias significativas en el paisaje bosque nativo (difieren los subgrupos botánicos y subgrupo turismo) y salto del Laja (entre el subgrupo turismo respecto a los subgrupos biólogos y botánicos). En la Tabla 3 se muestra un resumen de las principales características de las evaluaciones sobre cada macrounidad de paisaje.

\section{Variabilidad intergrupo de los observadores}

Según se observa en la Fig. 1 y la Tabla 4 existe una alta coherencia interna, entendida como una baja dispersión relativa de las ponderaciones, en los tres grupos, siendo algo mayor en el grupo control (promedio $=13,1 ; \mathrm{DE}=3,2 ; \mathrm{CV}=24,4 \%$ ), seguido del grupo exigentes (promedio $=11,6 ; \mathrm{DE}=3,4$ $\mathrm{CV}=29,3 \%$ ) y del grupo transformadores (promedio $=13,7 ; \mathrm{DE}=4,2 ; \mathrm{CV}=30,7 \%$ ). Esta coherencia interna relativa, de cada grupo, es más notoria frente a ciertos paisajes como la macrounidad bosque nativo en los grupos control $(\mathrm{CV}=17,1)$, exigentes $(\mathrm{CV}=21,9)$ y transformadores $(\mathrm{CV}=22,5)$.

En la Fig. 1 se aprecia que los tres grupos de observadores evaluaron, globalmente, de forma 
TABLA 2

Variación de la evaluación del paisaje en el grupo de transformadores en un transecto (Cabrero-Puerto Montt) del sur de Chile. 1997. $\mathrm{B}=$ bosque nativo, $\mathrm{C}=$ cultivos, $\mathrm{P}=$ plantaciones, $\mathrm{RC}=$ río Cautín, $\mathrm{SL}=$ salto del Laja y $\mathrm{O}=$ obstrucciones. $*=$ Diferencias significativas al $5 \%, \mathrm{~ns}=$ sin diferencias significativas

Variation of the landscape evaluation in the transformer group in a Southern Chilean transect (Cabrero-Puerto

Montt). 1997. $B=$ native forest,$C=$ agriculture, $P=$ plantation, $R C=$ Cautín river, $S L=L a j a ~ F a l l ~ a n d ~ O=$ obstructions. $*=$ significative differences at $5 \%$, ns= no significative differences

\begin{tabular}{|c|c|c|c|c|c|c|c|c|c|c|c|c|c|}
\hline & & \multicolumn{6}{|c|}{ Ingenieros Agrónomos } & \multicolumn{6}{|c|}{ Médicos Veterinarios } \\
\hline & & $\mathrm{B}$ & $\mathrm{C}$ & $\mathbf{P}$ & $\mathrm{RC}$ & SL & $\mathrm{O}$ & B & $\mathrm{C}$ & $\mathrm{P}$ & $\mathrm{RC}$ & SL & $\mathrm{O}$ \\
\hline & B & ns & & & & & & $*$ & & & & & \\
\hline & $\mathrm{C}$ & & $*$ & & & & & & * & & & & \\
\hline Ingenieros & $\mathrm{P}$ & & & $*$ & & & & & & $*$ & & & \\
\hline \multirow[t]{5}{*}{ Forestales } & $\mathrm{RC}$ & & & & ns & & & & & & ns & & \\
\hline & SL & & & & & ns & & & & & & ns & \\
\hline & $\mathrm{O}$ & & & & & & ns & & & & & & ns \\
\hline & $\mathrm{B}$ & & & & & & & ns & & & & & \\
\hline & $\mathrm{C}$ & & & & & & & & ns & & & & \\
\hline Ingenieros & $\mathrm{P}$ & & & $r^{\prime}$ & & & & & & ns & & & \\
\hline \multirow[t]{3}{*}{ Agrónomos } & $\mathrm{RC}$ & & & & & & & & & & ns & & \\
\hline & SL & & & & & & & & & & & ns & \\
\hline & $\mathrm{O}$ & & & & & & & & & & & & $\mathrm{ns}$ \\
\hline
\end{tabular}

similar la totalidad de los paisajes. Quienes evaluaron con mayores guarismos fue el grupo de transformadores (promedio $=12,5 ; \mathrm{DE}=4,7 ; \mathrm{CV}$ $=37,6)$, seguido por el panel control $($ promedio $=$ $11,9 ; \mathrm{DE}=4,7 ; \mathrm{CV}=39,5)$ y el de exigentes ( promedio $=10,6 ; \mathrm{DE}=4,0 ; \mathrm{CV}=37,7$ ).

No obstante lo anterior, al analizar las evaluaciones, por grupo, de las macrounidades de paisaje (Tabla 5) se aprecian diferencias significativas para algunas macrounidades. En general los gru- pos control y exigentes perciben de manera significativamente distinta los paisajes. Entre ellos se detectaron diferencias estadísticamente significativas al 95\%, sólo en las macrounidades de bosque nativo y salto del Laja y al $99 \%$, además, para salto del Laja (Tabla 5).

El caso de los paisajes de río Cautín ameritan un análisis más particular, ya que no es claro que las diferencias sean significativas (o que no lo sean). Para determinar qué grupos evaluaron de distinta

\section{TABLA 3}

Variación de la evaluación del paisaje en el grupo de exigentes en un transecto (CabreroPuerto Montt) del sur de Chile. 1997. DE= Desviación Estándar. CV= Coeficiente de Variación

Variation of the landscape evaluation in the demand group in a Southern Chilean transect (Cabrero-Puerto Montt). 1997. $\mathrm{DE}=$ Standard Deviation, $\mathrm{CV}=$ Coeficcient of variation

\begin{tabular}{|c|c|c|c|c|c|c|c|c|c|}
\hline \multirow[b]{2}{*}{ Macrounidad } & \multicolumn{3}{|c|}{ Biólogos } & \multicolumn{3}{|c|}{ Botánicos } & \multicolumn{3}{|c|}{$\begin{array}{c}\text { Profesionales } \\
\text { de Turismo }\end{array}$} \\
\hline & Promedio & $\mathrm{DE}$ & $\mathrm{CV}$ & Promedio & $\mathrm{DE}$ & $\mathrm{CV}$ & Promedio & $\mathrm{DE}$ & $\mathrm{CV}$ \\
\hline Bosque Nativo & 13,3 & 3,7 & 27,8 & 15,2 & 2,5 & 16,4 & 12,6 & 2,1 & 16,7 \\
\hline Cultivos Agrícolas & 10,1 & 3,4 & 34,7 & 11,9 & 3,8 & 31,9 & 11,7 & 2,6 & 22,2 \\
\hline Plantaciones Exóticas & 7,2 & 2,7 & 37,5 & 7,4 & 2,9 & 39,2 & 9,3 & 3,6 & 38,7 \\
\hline Río Cautín & 9,3 & 1,9 & 20,4 & 10,0 & 2,2 & 22,0 & 12,9 & 2,6 & 20,2 \\
\hline Salto del Laja & 17,1 & 7,4 & 43,3 & 17,0 & 5,4 & 31,8 & 21,1 & 3,1 & 14,7 \\
\hline Obstrucciones & 9,0 & 3,2 & 35,6 & 9,1 & 3,5 & 38,5 & 8,6 & 3,0 & 34,9 \\
\hline TOTAL PAISAJES & 11,0 & 3,7 & 33,6 & 11,8 & 3,4 & 28,8 & 12,7 & 2,8 & 22,1 \\
\hline
\end{tabular}




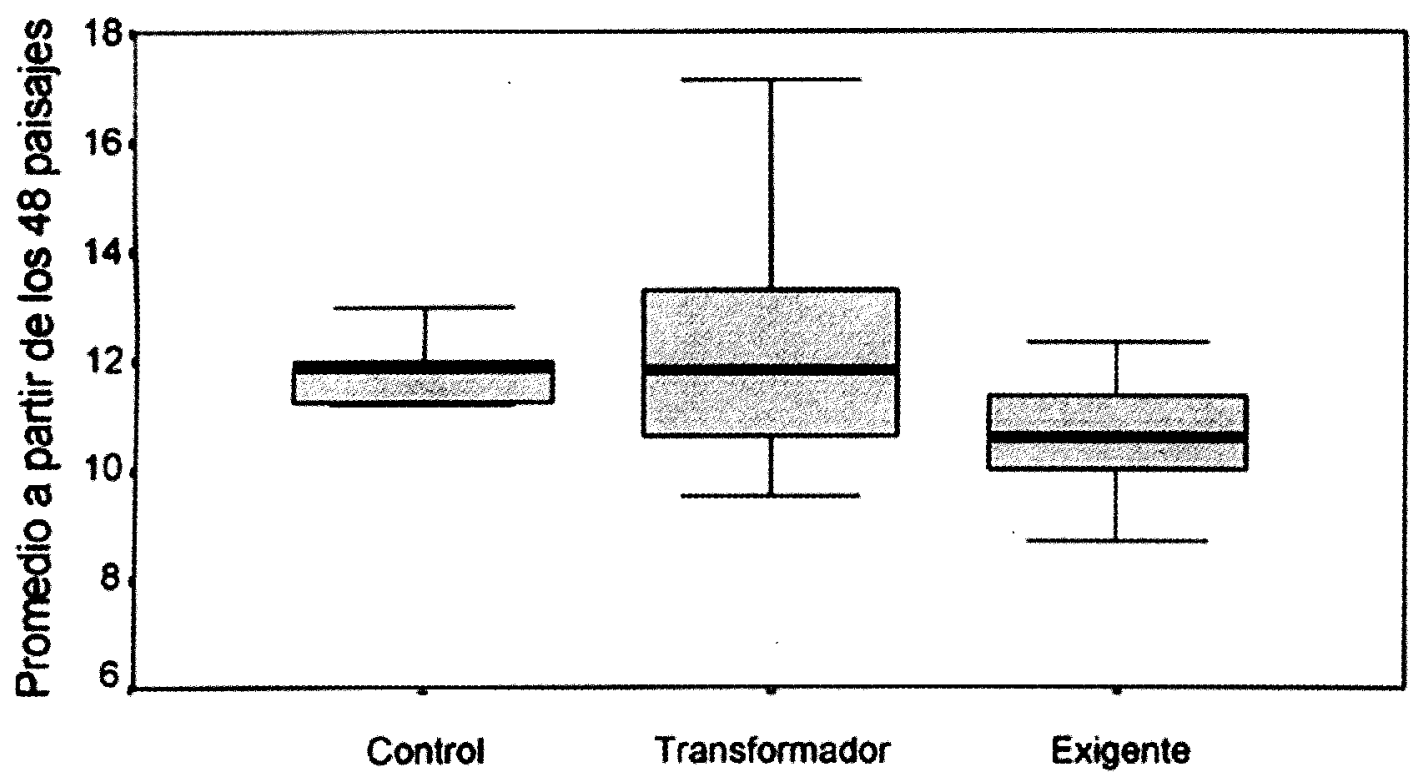

Fig. 1. Boxplot de tres grupos de evaluadores de paisajes en un transecto (Cabrero-Puerto Montt) del sur de Chile. 1997. Línea horizontal= mediana, Límite caja= cuartiles, Línea vertical acotada= extremos.

Boxplot of three landscapes evaluator groups in a Southern Chile transect (Cabrero-Puerto Montt). 1997. Horizontal line= mediane, Box limit= quartils, Acoted vertical line= extremes.

forma las macrounidades se sometieron los datos a las pruebas de Tukey y Scheffé las que establecieron que las diferencias significativas en la macrounidad río Cautín fueron entre los grupos transformadores y exigentes, en el caso de salto del Laja el grupo exigente evaluó de diferente forma tanto respecto al grupo transformadores como al control, no detectándose diferencias entre estos últimos (Tabla 6), y los paisajes de bosque nativo fueron percibidos como diferentes por transformadores y exigentes.

\section{Agrupamientos y constitución de paneles}

El comportamiento de los evaluadores frente a la macrounidad bosque nativo, se muestra en la Fig. 2 ; en la que se aprecia la existencia de dos grupos. Uno que se acerca a lo esperable del grupo control y otro hacia el grupo exigente. De este modo para evaluar bosque nativo son indispensables estos dos grupos, sin embargo no aporta mayor consistencia, a la evaluación, incluir un grupo de transformadores. Para el caso de las otras macro-

TABLA 4

Evaluación de paisajes de tres grupos de observadores en un transecto (Cabrero-Puerto Montt) del sur de Chile. 1997. $\mathrm{DE}=$ Desviación Estándar, $\mathrm{N}=$ número de paisaje contenidos en la macrounidad

Landscape evaluation of three observer group in a Southern Chilean transect (Cabrero-Puerto Montt). 1997. DE= Standard Deviation, = Number of landscape contents in the macrounit

\begin{tabular}{|c|c|c|c|c|c|c|c|c|c|c|}
\hline \multirow[b]{2}{*}{ Macrounidad } & \multicolumn{4}{|c|}{ Transformadores } & \multicolumn{3}{|c|}{ Exigentes } & \multicolumn{3}{|c|}{ Control } \\
\hline & $\mathbf{N}$ & Promedio & $\mathrm{DE}$ & $\mathrm{CV}$ & Promedio & $\mathrm{DE}$ & $\mathrm{CV}$ & Promedio & $\mathrm{DE}$ & $\mathrm{CV}$ \\
\hline Bosque Nativo & 30 & 16,0 & 3,6 & 22,5 & 13,7 & 3,0 & 21,9 & 15,8 & 2,7 & 17,1 \\
\hline Cultivos Agrícolas & 36 & 12,2 & 4,5 & 36,9 & 10,9 & 3,4 & 31,2 & 11,8 & 4,0 & 33,9 \\
\hline Plantaciones Exóticas & 36 & 9,1 & 3,2 & 35,2 & 7,7 & 2,9 & 37,7 & 7,6 & 2,8 & 36,8 \\
\hline Río Cautín & 9 & 13,9 & 3,4 & 24,5 & 10,2 & 2,1 & 20,6 & 11,8 & 2,6 & 22,0 \\
\hline Salto del Laja & 6 & 21,1 & 5,9 & 28,0 & 17,9 & 6,0 & 33,5 & 21,7 & 3,9 & 18,0 \\
\hline Obstrucciones & 15 & 10,0 & 4,3 & 43,0 & 8,9 & 3,2 & 36,0 & 9,7 & 2,9 & 29,9 \\
\hline Total paisajes & 132 & 13,7 & 4,2 & 30,7 & 11,6 & 3,4 & 29,3 & 13,1 & 3,2 & 24,4 \\
\hline
\end{tabular}


TABLA 5

Comparación de evaluaciones promedio de tres grupos de observadores en un transecto (Cabrero-Puerto Montt) del sur de Chile. 1997. F= Cuociente de Fisher, p= significancia estadística, ${ }^{*}=$ diferencias significativas al $99 \%, * *=$ diferencias significativas al $95 \%$

Evaluation comparation of three observer groups in a Southern Chilean transect (Cabrero-Puerto Montt). 1997. F= Fisher rate, $p=$ statistical significance, $*=$ significative differences at $99 \%, * *=$ statistical significance at $95 \%$

\begin{tabular}{|c|c|c|c|c|c|c|}
\hline Macrounidad & $\begin{array}{c}\text { Promedio } \\
\text { evaluaciones } \\
\text { transformadores }\end{array}$ & $\begin{array}{c}\text { Promedio } \\
\text { evaluaciones } \\
\text { exigentes }\end{array}$ & $\begin{array}{c}\text { Promedio } \\
\text { evaluaciones } \\
\text { control }\end{array}$ & F & $\mathrm{p}$ & \\
\hline Bosque Nativo & 16,0 & 13,7 & 15,8 & 4,98 & 0,012 & $(*)$ \\
\hline Cultivos Agrícolas & 12,2 & 10,9 & 11,8 & 1,23 & 0,304 & \\
\hline Plantaciones Exóticas & 9,1 & 7,7 & 7,6 & 1,43 & 0,252 & \\
\hline Río Cautín & 13,9 & 10,2 & 11,8 & 3,05 & 0,059 & $(*)$ \\
\hline Salto del Laja & 21,1 & 17,9 & 21,7 & 7,49 & 0,001 & $(* *)$ \\
\hline Obstrucciones & 10,0 & 8,9 & 9,7 & 1,38 & 0,264 & \\
\hline
\end{tabular}

unidades el análisis no es concluyente, por lo que para evaluar estos paisajes (e.g., cultivos, plantaciones) se deben considerar los tres grupos.

Los Ingenieros Forestales percibieron de modo muy diferente los paisajes generados por las actividades silvoagropecuarias respecto a veterinarios y agrónomos. Estos últimos sólo se acercan a los forestales, en sus preferencias, cuando evalúan el bosque nativo. Respecto a plantaciones es notoria la mayor valoración de los forestales, así como de los cultivos por parte de los agrónomos. Es claro que existe una preferencia, vinculada con la actividad profesional, por lo que la primera recomendación, al constituir un panel de evaluadores transformadores, es tener en cuenta estas variaciones. En general la incorporación de forestales se hace indispensable a la hora de evaluar plantaciones artificiales. La composición de

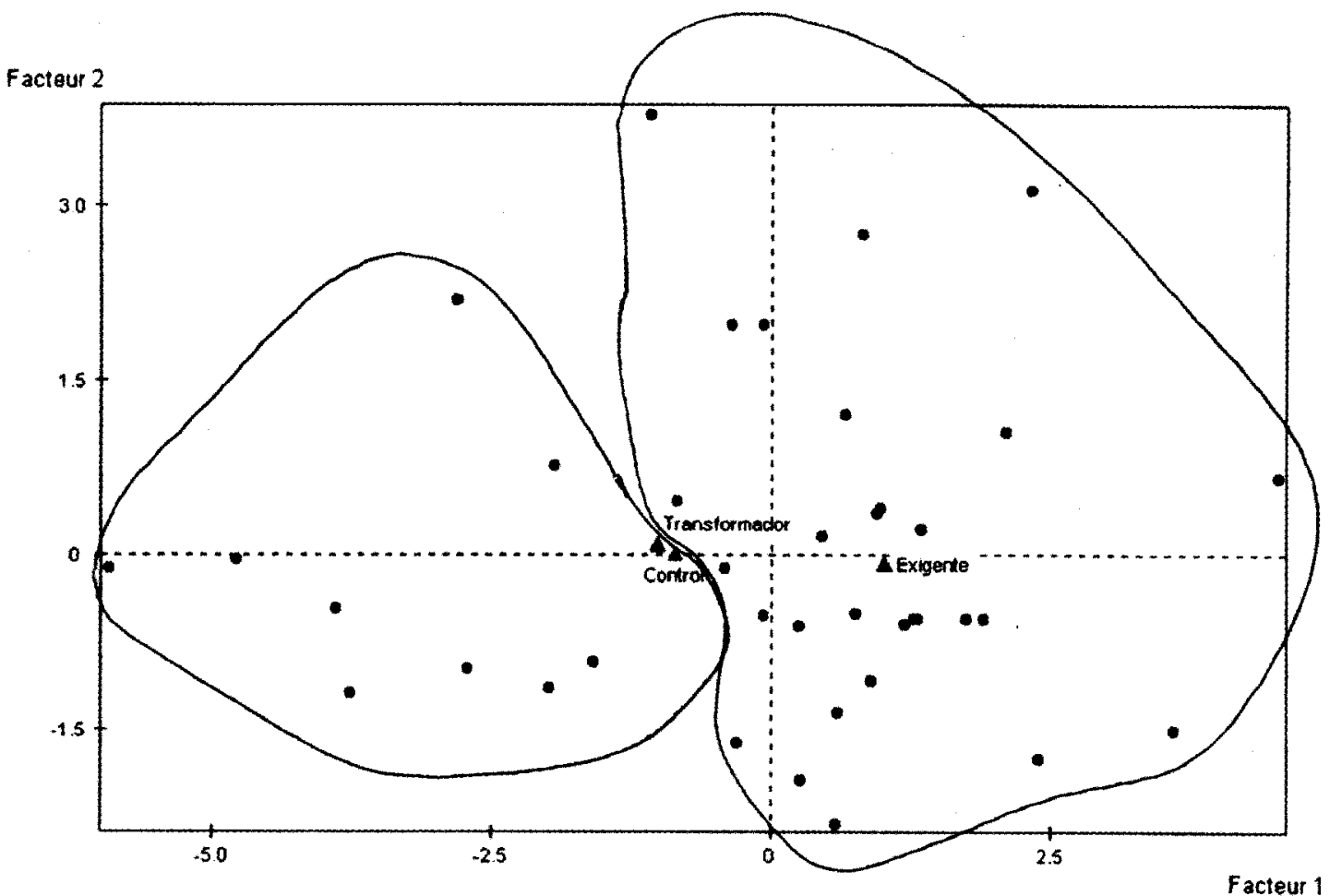

Fig. 2. Representación factorial del agrupamiento de los evaluadores frente a la macrounidad bosque nativo.

Factorial representation of the evaluator groups facing the native forest macrounit. 
Comparación de similitud y diferencias, según las pruebas de Tukey y Scheffé, en las evaluaciones de tres grupos de observadores en un transecto (Cabrero-Puerto Montt) del sur de Chile. 1997. Evaluaciones verticales, bajo las X, denotan similitud en la evaluación de los grupos

Comparision of the similarity and differences comparation, according to Tukey and Scheffé tests, in the evaluation of three group of observator in a Southern Chilean transect (Cabrero-Puerto Montt). 1997. Vertical Evaluation, under the $\mathrm{x}$ denoted similarity in the evaluation group

\begin{tabular}{lcccccc}
\hline & Bosque & $\begin{array}{c}\text { Cultivos } \\
\text { nativo }\end{array}$ & Plantaciones & $\begin{array}{c}\text { Río } \\
\text { exóticas }\end{array}$ & $\begin{array}{c}\text { Salto } \\
\text { Cautín }\end{array}$ & $\begin{array}{c}\text { Obstrucciones } \\
\text { Del Laja }\end{array}$ \\
\hline $\begin{array}{l}\text { Grupo } \\
\text { Control }\end{array}$ & $\mathrm{X} \mathrm{X}$ & $\mathrm{X}$ & $\mathrm{X}$ & $\mathrm{X} \mathrm{X}$ & $\mathrm{X}$ & $\mathrm{X}$ \\
$\begin{array}{l}\text { Grupo } \\
\text { Transformadores }\end{array}$ & $\mathrm{X}$ & $\mathrm{X}$ & $\mathrm{X}$ & $\mathrm{X}$ & $\mathrm{X}$ & $\mathrm{X}$ \\
$\begin{array}{l}\text { Grupo } \\
\text { Exigentes }\end{array}$ & $\mathrm{X}$ & $\mathrm{X}$ & $\mathrm{X}$ & $\mathrm{X}$ & & $\mathrm{X}$ \\
\hline
\end{tabular}

este grupo de transformadores, sin embargo, no es relevante si son paisajes no vinculados a las actividades silvoagropecuarias, como paisajes lacustres, ribereños (A. Muñoz-Pedreros datos no publicados).

Los evaluadores exigentes (e.g., botánicos), 10 son en forma particular con los paisajes antropizados, 1o que puede explicarse por las características formativas de este grupo. MuñozPedreros et al. (1993) demostraron que los estudiantes universitarios de turismo son observadores exigentes, por su formación profesional, no así los de ingeniería, que están fuertemente condicionados por el medio construido y son menos exigentes en paisajes naturales. Por otro lado, los profesionales vinculados a actividades transformadoras del paisajes son menos exigentes, como los profesionales silvoagropecuarios (esta investigación) y los vinculađos a la ingeniería civil (Muñoz-Pedreros et al. 1993). De esta forma la segunda recomendación es constituir un panel con tres grupos representativos: transformadores, exigentes y control. Por motivos operacionales y de costos recomendamos un mínimo de cinco panelistas por grupo.

Muñoz-Pedreros et al. (1993) probaron que para unidades de paisaje en el sur de Chile la procedencia y el sexo de los evaluadores no intervinieron en la apreciación y evaluación de los paisajes, pero sí la edad. Como se espera que los procedimientos aqui estudiados sean empleados en herramientas de gestión ambiental (e.g., estudios de Impacto Ambiental) se propone excluir a personas menores de 18 años o pertenecientes a la tercera edad, ya que se sugiere que los paneles de evaluadores esten constituídos por profesionales que realizen un trabajo técnico y normado. Benayas (1992) apoya la idea de que el ser humano, en una edad periférica a la adolescencia tiende a modificar secuencialmente sus actitudes con respecto al entorno. A modo de ejemplo, en experiencias con niños españoles, probaron que la valoración de paisajes que contenían animales domésticos, varió con la edad y así los niños mayores preferían animales más bien silvestres o salvajes (Múgica et al. 1989²). Existe variabilidad frente al menos dos tipos de paisaje: los antropizados (ordenados, ajardinados y/o cultivados) y los naturales (más salvajes, vírgenes o espontáneos). Los primeros son percibidos como paisajes controlables, confortables y seguros. En cambio los segundos son paisajes percibidos como más estimulantes, por explorar y sugerentes. Los paisajes antropizados son, en general preferidos por niños hasta los 15 años y por adultos mayores de 35, así como por mujeres, en cambio los paisajes más naturales son preferidos por jóvenes de 15 a 35 de preferencia hombres. Los recuerdos más gratos parecieran provenir de ambientes antrópicamente no estructurados (Roberto Lira comunicación personal). En conclusión, y como se pretende desarrollar un procedimiento para la toma de decisiones respecto al recurso, la tercera recomendación es la constitución de los paneles con personas mayores de edad (e.g., > 21 años).

${ }^{2}$ MUGICA M, J BENAYAS, JP RUIZ, FG BERNALDEZ \& JV DE LUCIO (1989) Analysis of children's attitudes towards animals. V International Conference on the relationship between human and animals. 
Benayas (1992) encontró que los universitarios por él estudiados, que tienden a preferir los paisajes más autóctonos, equilibrados y con pocos impactos humanos, presentan unos esquemas cognitivos de interpretación del paisaje más complejos y diversos. Esto es coherente con Fines (1968) que demostró, en el procedimiento de valoración del paisaje con fotografías, la importancia del aprendizaje y la experiencia en su apreciación, constituyéndose en la única forma de evitar influencias emocionales y educativas o del gusto personal (Gómez 1980). Muñoz-Pedreros et al. (1993) también probaron que el nivel de conocimiento del área evaluada influye en la evaluación, por lo que se deben excluír evaluadores que conozcan las imagenes a evaluar, para evitar valoraciones sesgadas por recuerdos y asociaciones. De este modo un cuarta recomendación es excluir a personas que ya tengan conocimientos de los paisajes a evaluar. Respecto a la participación de estudiantes de educación superior, varios autores los han empleado y han resultado ser representativos de los grupos poblacionales (véase Brown \& Daniels 1986).

El control de la subjetividad en la evaluación de los paisajes debiera aumentar. Fines (1968) argumenta que la escala universal de valores por él propuesta será unánime a medida que mejoren los estándares de educación y el contacto con el sistema natural (véase para otras críticas y defensas CEOTMA1982, Penning-Rowsell 1974).

\section{CONCLUSIONES}

Los grupos de evaluadores difieren en sus preferencias frente a los paisajes, lo que se puede explicar por las distintas formaciones profesionales de los evaluadores. Por otro lado existe coherencia entre los grupos y características importantes del paisaje que son apreciados en forma unánime (e.g., bosques nativos). La evaluación hecha con un panel según las recomendaciones propuestas, puede ofrecer una razonable objetividad, que permita comparar paisajes, cuantificar calidades y tomar decisiones en la gestión del recurso paisaje.

\section{AGRADECIMIENTOS}

Los autores agradecen a los proyectos DIUCT 94-4 y DIUCT 97-4-01 de la Dirección de Investigación de la Universidad Católica de Temuco y al proyecto P.I. 95.310.020-6 de la Dirección de Investigación de la Universidad de Concepción. También agradecen a Jaime Rau por sus valiosos comentarios críticos al manuscrito. Esta investigación es parte de la tesis doctoral del primer autor (Doctorado en Ciencias Ambientales del Centro EULA, Universidad de Concepción).

\section{LITERATURA CITADA}

APPLETON J ed (1981) The aesthetics of landscape. Proceedings of a Symposium held. En: the University of Hull. Rural Planning Service. U.K.

BENAYAS J (1992) Paisaje y Educación Ambiental, evaluación de cambios de actitudes hacia el entorno. Monografías de la Secretaría de Estado para las Políticas del Agua y el Medio Ambiente. Ministerio de Obras Públicas y Transportes, Madrid. $243 \mathrm{pp}$.

BENAYAS J (1994) La percepción del paisaje. En: Benayas J, F Heras, JV Lucio, C Marcen, E Pino \& JP Ruiz (eds) Viviendo el Paisaje. Guía didáctica para interpretar y actuar sobre el paisaje: 19-25. Fundación NatWest, Fundación para la Investigación y el Desarrollo Ambiental, Madrid.

BERNALDEZ FG (1981) Ecología y Paisaje. Editorial H Blume, Madrid. $256 \mathrm{pp}$.

CEOTMA (1982) Guía para la elaboración de estudios del Medio Físico. Contenido y metodologías. Centro de Estudios de ordenación del territorio y medio ambiente MOPU. Serie Manuales $N^{\circ} 3$ Madrid, España. $572 \mathrm{pp}$.

CRAIK KH (1975) Individual Variations in Landscapes Description. En: Zube EH, RO Brush \& JG Fabos (eds) Landscape Assessment, Values Perceptions and Resources: 130-150. Dowden, Hutchinson \& Ross Inc. Stroudsburg, Pennsylvania.

COLLINS A (1992) Introduction to Multivariate Analysis. Chapman and Hall Cambridge, Great Britain. 246 pp.

CRIVISQUI E (1993) Análisis Factorial de Correspondencias. Editorial de la Universidad Católica de Asunción. Asunción, Paraguay. 302pp.

DANIEL TC \& RS BOSTER (1976) Measuring landscape esthetics: The Scebic Beauty Estimation method. USDA Forest Service Research Paper RM-167. 66 Pp.

DE VEER AA \& PA BURROGH (1978) Physiognomic Landscape Mapping in the Netherlands. Landscape Planning 5:45-62.

DUNN MC (1974) Landscape Evaluation Techniques: An Appraisal and Review of the Literature. Centre for Urban and Regional Studies, University of Birminghan. $255 \mathrm{pp}$.

ESCRIBANO MM, M DE FRUTOS, E IGLESIAS, C MATAIX \& I TORRECILLA (1991) El Paisaje. Ministerio de Obras Púbicas y Transportes de España. $117 \mathrm{pp}$.

FINES KD (1968) Landscape evaluation: a research proyect in East Sussex. Regional Studies (UK). 2: 41-55.

GALIANO \& ABELLO (1984) Una metodología para la valoración del paisaje en estudios de ordenación territorial. Su aplicación al Término municipal de la Granja de San Ildefonso. Ciudad y Territorio, julio/ septiembre (España): 53-58. 
GOMEZ D (1980) El Medio Físico y la Planificación . Cuadernos del Centro Internacional de Formación en Ciencias Ambientales (Madrid) 10-11: 1-299.

JACKSON (1978) Assessment of the environmental impact of high voltage Power Transmission Lines. Journal of Environmental Management 6: 153-170.

JACQUES DL (1980) Landscape appraisal: the case for a subjetive theory. Journal of Environmental Management 10: 107-113.

JOHNSON-WICHERN (1988) Applied Multivariate Statitical Analysis. Second edition. Prentice-Hall International Editions, N.J., U.S.A. 607 pp.

KAPLAN S (1976) Adaptation, structure and knowledge. En: Moore GT \& R Golledge (eds) Environmental Knowing: theories, research and methods: 3-25.

KAPLAN S \& R KAPLAN (1982) Cognition and Environment. Preager, New York. 125 pp.

LITTON B (1972) Aesthetic Dimensions of the Landscape In Natural Environments Studies in Theoretical an Applied Analisis. En: Krutilla J (ed) Resources for the Future: 262-291.The Johns Hopkins University Press, Baltimore.

LUCIO JV (1994) La diversidad paisajistica. En: Benayas J, F Heras, JV Lucio, C Marcen, E Pino \& JP Ruiz (eds) Viviendo el Paisaje. Guía didáctica para interpretar y actuar sobre el paisaje: 27-36. Fundación NatWest, Fundación para la Investigación y el Desarrollo Ambiental, Madrid.
MOPT (1993) Guía Metodológica para el estudio del Medio Físico y la Planificación. Ministerio de Obras Públicas y Transporte, series monográficas, Madrid. $809 \mathrm{pp}$.

MUÑOZ-PEDREROS A, A BADILLA \& H RIVAS (1993) Evaluación del paisaje en un humedal del sur de Chile: el caso del río Valdivia (X Región). Revista Chilena de Historia Natural 66: 403-118.

RAMIREZ C, C SAN MARTIN \& R MAC DONALD (1992) El paisaje vegetal como indicador de cambios ambientales. Ambiente y Desarrollo (Chile) : 67-71.

SAVOLAINEN R \& S KELLOMAKI (1984) Scenic value of the forest landscape as assessed in the field and the laboratory. En: Multiple-use forestry in the Scandinavian countries: 73-80. Forestalia Fennica Helsinski, Finland.

WEDDLE AE (1973) Applied analysis and evaluation techniques. En: Lovejoy D (èd) Land use and Landscape Planning: 53-82. Leonard Hill Brooks, the Pittman Press, Bath.

Editor Asociado E Hajek

Recibido el 30 de noviembre de 1999; aceptado el 7 de julio de 2000 